\title{
Inherently Bio-Active Scaffolds: Intelligent Constructs to Model the Stem Cell Niche
}

\author{
Paolo Di Nardo, Marilena Minieri, Annalisa Tirella, \\ Giancarlo Forte and Arti Ahluwalia
}

\begin{abstract}
The oft-abused phrase "genes load the gun, environment pulls the trigger" can be applied to stem cells and stem cell niches as well as to cellmaterial interfaces. Much is known about cell-material interaction in general, perhaps a little less about how these interactions condition cell phenotype. With the increasing interest in stem cells and, in particular, their applications in tissue regeneration, the regulation of the stem cell microenvironment through modulation of intuitive or smart materials and structures, or what we term IBAS (Inherently Bio-Active Scaffolds) is poised to become a major field of research. Here, we discuss how cardiac regeneration strategies have undergone a gradual shift from the emphasis on biochemical signals and basic biology to one in which the material or scaffold plays a major role in establishing an equilibrium state. From being a constant battle or tug-of-war between the cells and synthetic environments, we conceive IBAS as intuitively responding to the cell's requirements to instate a sort of equilibrium in the system.
\end{abstract}

\footnotetext{
P. D. Nardo $(\bowtie) \cdot$ M. Minieri · A. Tirella · G. Forte

Laboratorio di Cardiologia Molecolare e Cellulare, Dipartimento di Medicina Interna, Università di Roma Tor Vergata, Via Montpellier, 1, 00133 Rome, Italy

e-mail: dinardo@uniroma2.it

P. D. Nardo · M. Minieri - G. Forte

Istituto Nazionale per le Ricerche Cardiovascolari (INRC), Bologna, Italy

P. D. Nardo - M. Minieri · G. Forte

Japanese-Italian Tissue Engineering Laboratory (JITEL), Tokyo Women's Medical University-Waseda University Joint Institution for Advanced Biomedical Sciences

(TWIns), Tokyo, Japan
}

A. Tirella · A. Ahluwalia

Centro Interdipartimentale di Ricerca “E. Piaggio”, Università di Pisa, Pisa, Italy 


\section{Introduction}

The heart, with its romantic associations as well as its fundamental role as the machine of human life, is one of the most well-known and studied organs in the body. But its failure or damage still causes the highest number of deaths per year in developed countries. Indeed, the conventional clinical approach and treatment (early diagnosis + drugs) has failed so far in curing heart failure, a complex pathophysiological state in which the organ contractile activity is no longer able to deliver an adequate quantity of blood and nutrients to match tissue requirements. Furthermore, heart transplants, given the lack of donors and the low costeffectiveness, is an option open to a tiny percentage of patients. For these reasons, cardiomyocyte regeneration through the use of stem cells has received unparalleled attention both in the press and within the professional academic and medical fields. However, there is a desperate lack of fundamental knowledge on cell regeneration in general, and cardiac tissue regeneration in particular. Our understanding of cardiomyocyte origin and potency is limited; there is a huge amount of controversy and mis-information regarding cardiac stem cells, their isolation, their identification and their expansion. Moreover, despite decades of experimental research on biomaterials and scaffolds, neither the ideal material nor the architecture for cardiac cell proliferation and colonization has been identified.

In 1994, the possibility of regenerating the heart was thoroughly discussed, for the first time, in a Congress in Viterbo (Italy) that gathered the most prominent international scientists of that time [14], but the technology was not adequate to start experimentation and envisage clinical applications. Nevertheless, after a few years, advancements in cell biology and material sciences provided the impetus to start preliminary experiments, in which mostly mature cardiomyocytes were: (i) injected into damaged experimental animal and human heart walls or (ii) used to fabricate very rudimentary parts of myocardium. This approach, as a whole, was unsuccessful. Scientists thus turned their attention to stem cells but, once again, the injection of different stem cell populations into damaged myocardium was inefficient. Many researchers proposed fabricating ex vivo portions of myocardium to be engrafted into injured hearts using a biomaterial support on which stem cells can be grown. Attention was thus drawn to biocompatible materials already in use in the clinical setting, and to the identification of reliable and well-characterized sources of cardiogenic stem cells.

Numerous experiments and options have since been evaluated, in order to identify the best materials and the optimum technologies to design and process scaffolds. However, a consistent design strategy, and well-founded engineering principles and models have been sorely missing from the research scenario. Properly designed scaffolds should deliver signals perceived as "suitable" by stem cells and capable of mimicking the natural 3D environment of the heart. The design of smart biomaterials for tissue regeneration is critically dependent on the fundamental understanding of how cells coordinate their functions in the in vivo environment and in engineered matrices. Therefore, matrix/cell and scaffold/cell 
interactions must first be properly investigated through both numerical modeling and experimental assessment, and only then can they be validated in vitro and ultimately in vivo.

There is no doubt that current materials and methods cannot deliver functional heart tissue as required for cardiac regeneration, and a change in perspective and strategy is required. Therefore, it is necessary to stimulate the generation of novel biomimicking strategies for inducing cardiac self-repair by proposing and promoting new approaches both in material design and modeling strategies.

\section{Towards a Paradigm Shift}

Not surprisingly stem cell-based regeneration of the heart has elicited much scientific and public attention, but has also generated commercial interests. Currently, post-infarction myocardial revascularization protocols include the administration of mesenchymal stem cells, either by intravascular or intramyocardial injection. Although a number of controlled clinical trials have been performed, the results have so far been controversial [12, 23, 29, 77]. The small improvements reported have been mostly ascribed to stem cell paracrine effects or to their pro-angiogenic activity rather than to a direct contribution to cardiac muscle repair. Besides the fact that the types of cells suitable for heart regeneration in man remain to be defined [49, 53], it is clear that generating new cardiomyocytes may be not sufficient to efficiently repair the texture of myocardial tissue, which also includes fibroblasts, smooth muscle cells, endothelial cells and adipocytes, among others. In healthy human hearts, only $10-20 \%$ of the cell complex constituting the whole organ are cardiomyocytes $[56,68]$ and at the age of 25 years no more than $1 \%$ of them are annually substituted by progenitor cells, with the percentage falling to less than $0.5 \%$ at the age of 75 . In total, less than $50 \%$ of all cardiomyocytes are renewed during a normal human life span [7]. In addition, it is likely that there is a natural limitation to progenitor cell capability to repair injured myocardium, considering that infarction as well as widespread myocardial diseases progress to fibrosis and not to newly generated contractile tissue. Nevertheless, features so far documented in reliably isolated and manipulated progenitor cells resident within the cardiac tissue (cardiac progenitor cells, CPC), although fragmentary and often controversial, do suggest that innovative treatments to repair damaged myocardium could have promising clinical applications.

\section{Who and Where are the Stem Cells?}

Cardiac progenitor cells and bone-marrow-derived stem cells share antigenic markers. Thus, it has been suggested that bone marrow could be the source of all stem cell populations having mesenchymal-like features resident within 
different tissues [6]. However, CPC appear within the myocardium during ontogeny and remain quiescent within their niches for short or long periods of time [39]. However, in spite of this unifying view, various CPC classifications have been so far proposed and an unambiguous interpretation is still lacking. As shown in Table 1, a number of cell markers have been credited to identify CPC, but they are not exclusive and their expression pattern can diverge among species. In addition, the existence of progenitor cells self-organizing into cardiospheres has been challenged by [3], who have demonstrated that the cardiosphere cells are not cardiomyogenic and their apparently spontaneous beating is produced by contaminations from cardiac tissue. Therefore, at the present level of knowledge, the identification of the progenitor cells is only based on their capability to self-renew and to produce differentiated progeny [18].

In the past, most studies agreed that progenitor cells (single or clustered) are settled in specific anatomic and functional locations (niches), where cells are protected by depletion and, in turn, the host tissue by their over-proliferation. Niches mediate signals maintaining progenitor cell self renewal and multipotency or inducing their commitment in response to tissue specific needs [64]. To date, though, cardiac progenitor cell niches have never been exhaustively identified or described, very likely because they do not have anatomical dimensions in all organs $[25,82]$.

\section{Unstable Niches}

The stem cell niche is generally considered as an anatomic and functional unit, in which stem cells are confined in a semi-quiescent state. In the heart we hypothesize that niches have only functional and temporal dimensions and are characterized by a dynamically symmetric array of signals delivered by the neighboring microenvironment, as depicted in Fig. 1. In our opinion, the signal symmetry maintains progenitor cells in a metastable quiescent state, even for long periods of time, preventing aging processes and preserving their multipotency [4]. According to this interpretation, stem cells are inherently unstable (i.e., prone to chaotically adopt multiple phenotypes) and only a complex array of competing symmetric signals confines them in a metastable equilibrium (Fig. 2). A very small modification in local or long-range extracellular signaling can attract progenitor cells towards one of many possible stable states. All this occurs within a highly stable global context, the myocardium. Once the symmetry of signals maintaining the metastable equilibrium has been broken by internal and/or external factors, progenitor cells engage in differentiating pathways and tissue assembly processes in an ever-changing environment characterized by an array of qualitatively and quantitatively diverse factors (biochemical, physico-chemical and mechanostructural) arranged along the time axis to generate a dynamic multicomponent template of microenvironmental symmetry. The progenitor cell metastable state might biologically correspond to fluctuating levels of transcription factors with 


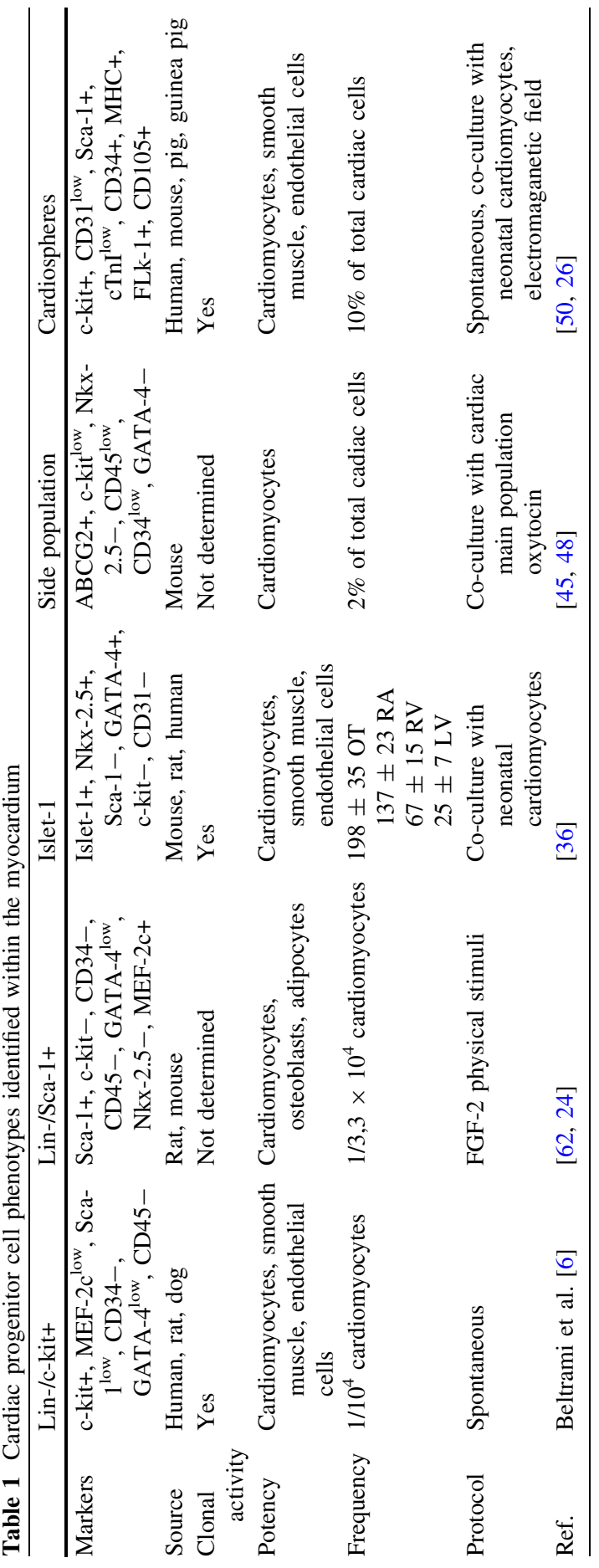


Fig. 1 The symmetric array of signals which contribute to defining the cardiac stem cell niche

Fig. 2 The unstable progenitor cells are in metastable equilibrium while confined in a niche featured by an appropriate signal symmetry. The evolution of the niche signal symmetry drives progenitor cell differentiation
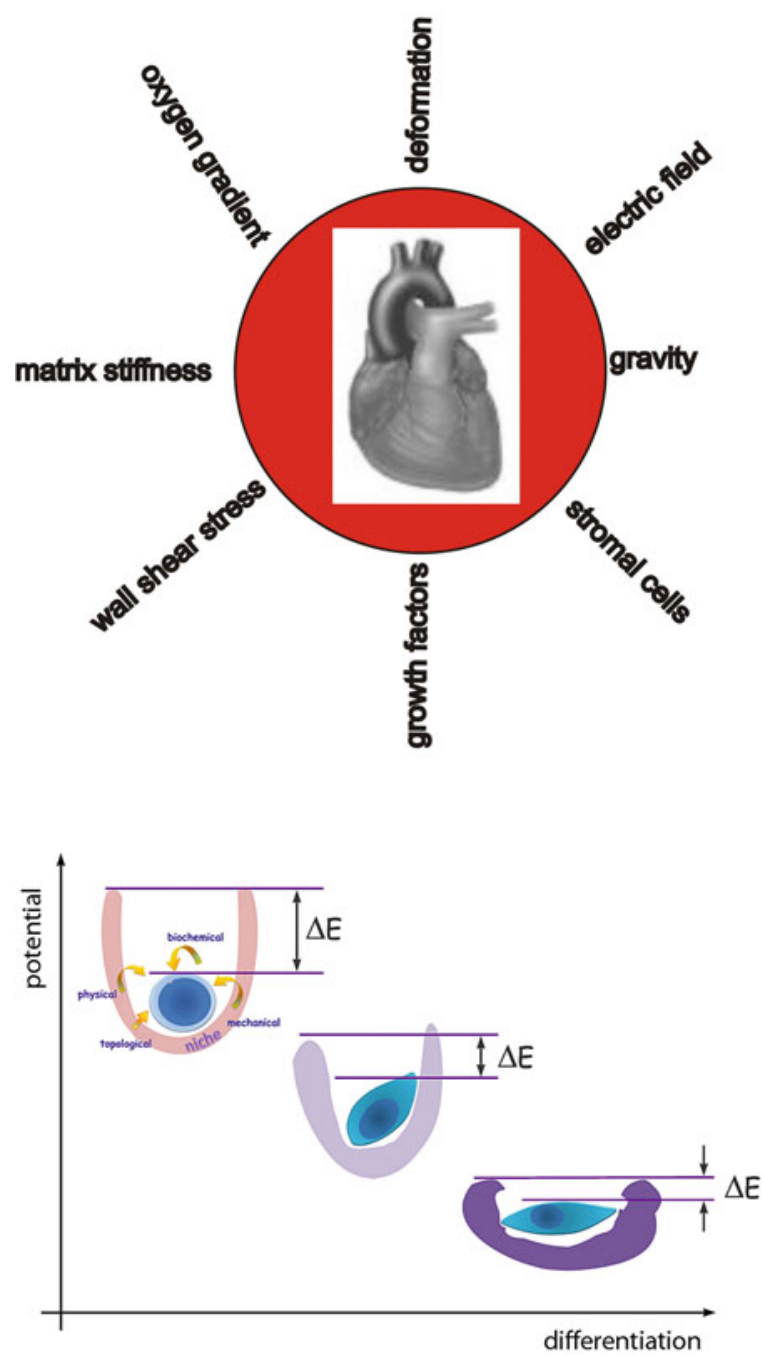

threshold-dependent commitment, similar to that described in the haemopoietic system [16]. This implies that niches are protempore activated loci, where the natural progenitor cell instability is confined (native niche) or is governed in favor of the acceptance of the required phenotype (differentiating niche) and tissue architecture (architectural niche). These different niches do not identify specific regions within the myocardium, but correspond to a continuum of different critical levels of interplay adopted by a wide array of multiparametric signals in response to local tissue requirements. We therefore prefer to think of them as states, or energy levels, as schematized in Fig. 2. Differentiating progenitor cells undergo a labile transition state, while migrating to niches characterized by a more stable, although more segregating, level of symmetry. This process occurs through 
environment-controlled differential segregation of progenitor cell "sensors" (membrane and intracellular proteins), so that only those specifically correlated with a definite step are active in differentiating progenitor cells.

The elusive goal we are all aiming to is to replicate this continuum of niches in vitro. Consistently, we need to shift from the emphasis on a trial and error based biochemical tuning of cardiac phenotype, towards more rigorous and well defined engineering and modeling of the stem cell microenvironment.

\section{Engineering Factors Which Determine Phenotype}

Cell function and fate are determined by three clusters of factors in the microenvironment: (i) the biochemical microenvironment, including ligands, signaling molecules and other cells, (ii) the physico-chemical environment, which comprises gradient-dependent factors, such as surface properties, oxygen tension, $\mathrm{pH}$ and temperature, and, finally, (iii) the mechano-structural environment. The mechanostructural environment is the architecture in 2 and 3 dimensions as well as mechanical forces, such as stress and strain, all of which act in a non-linear, but fairly constant manner. Together the three elements represent what we define as the tripartite axes of cues, as depicted in Fig. 3. Cell fate and function also depend strongly on time; even stem cells are equipped with a biological clock which ticks constantly.

Much of the information on cardiac stem cell biology to date has been obtained with respect to their responsiveness to a few soluble factors (growth factors/ cytokines, epigenetic factors, etc.) in vitro. In most cases, the signalling pathways involved in stem cell growth and differentiation are still not clearly identified and methods used to differentiate cells are determined empirically on the basis of trial and error. Till recently, the concept that stem cell fate is mostly governed by

Fig. 3 The three main groups of stimuli which condition the cell microenvironment. The sphere represents time, a constant and irreversible parameter which stretches out in all three dimensions

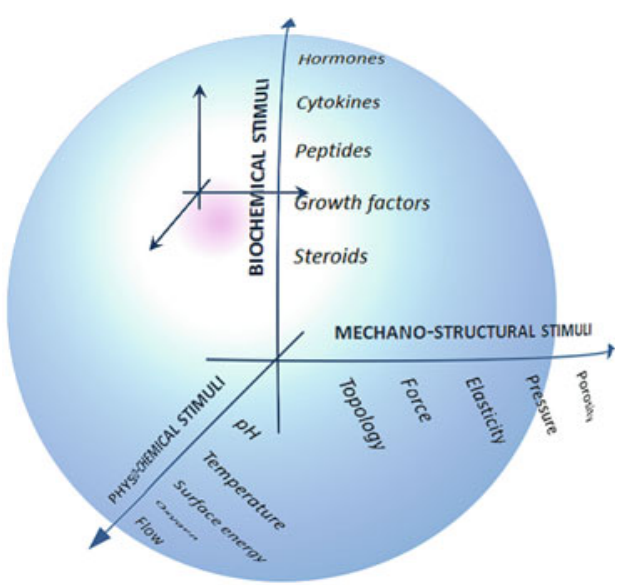


soluble factors has been passively accepted by investigators. As a consequence, all attempts at replicating in vitro the niche conditions and at activating both in vitro and in vivo stem cell lineage specification have been based on the assumption that a few soluble factors, sometimes injected in either the bloodstream or the damaged tissue, can govern the whole process leading stem cells to full differentiation, i.e. stem cell specification and determination, sarcomere assembly and cardiomyocyte integration into the myocardial architecture [17, 80]. This simplistic vision has been recently challenged by the demonstration that a pivotal role to accurately control cardiac stem cell fate in vitro is played by mechano-structural and physicochemical cues that, among others, are much easier to engineer [24, 61].

As represented in Fig. 3, the mechano-structural axis comprises those cues which condition the static nature of the cell habitat. Here, we include 3D architecture, surface physical features, such as roughness, as well as bulk properties, such as elastic modulus as well as stress, strain and force. For example, the most striking and repeatable differences are found between 2 and 3 dimensional (3D) environments. In fact, there is a dramatic change in habitat when cells are removed from an in vivo context to a petri dish, and in particular stem cells find themselves in a highly non-symmetrical context. Therefore, several reports have shown that encapsulated cells or spheroids are capable of maintaining stemness, and this could be because the microenvironment does not condition the cells to express differentiation proteins for coupling to an extracellular environment. Although there are few systematic studies on the comparative effects of 2 and 3D structures, there are clear indications that cells respond quite differently to the 3D environment which allows greater cell-cell interaction, and maintenance of spherical morphologies. In this context, hydrogels, such as those derived from alginate, collagen and hyaluronic acid, have been shown to be quite promising - they provide a homogeneous, structure-less soft 3D environment, which is probably ideal for stem cell proliferation and maintenance, as well as for differentiation into softer tissues, such as neural or hepatic $[5,10]$.

Three dimensional environments can also provide more controlled spatial information to cells, if they are architectured using techniques such as microfabrication based on rapid prototyping. In this case, the $3 \mathrm{D}$ system is usually nominated as a "scaffold", and provides a rigid and porous framework for cells to adhere on and spread in 3 dimensions. One of the first reports on architectured scaffolds employed for stem cell engineering describes a random pore $(250-500 \mu \mathrm{m})$ salt leached synthetic polymer scaffold (a blend of polylactide (PL) and polylactide-co-glycolide (PLGA) seeded with embryonic stem cells (ESC) [38]. The cells generated complex capillary-like organized features which cannot be formed in 2D. Moreover the porous scaffold permitted the ESC to organize and orient, whereas a homogeneous isotropic soft gel did not, suggesting that both stiffness and a structured topology are important features to which the cells respond.

Further studies by Liu and Roy [41, 42] also confirmed that a porous rigid scaffold (tantalum in this case) promotes differentiation of ESC into hematopoietic cells with respect to classical $2 \mathrm{D}$ cultures. Here, the cells were observed to interact 


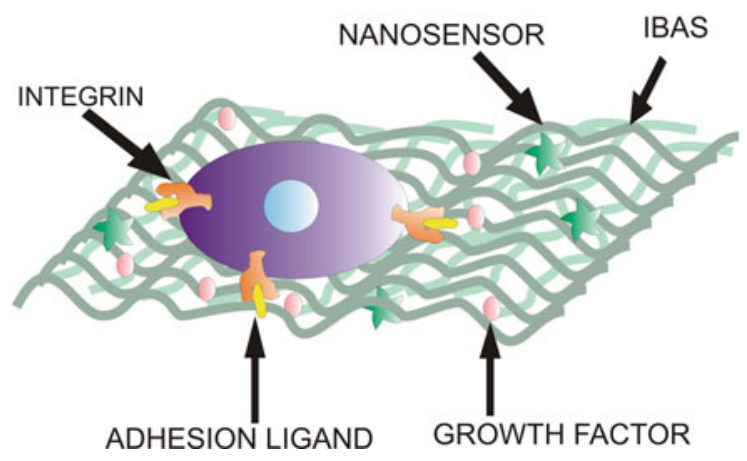

Fig. 4 IBAS (inherently bio-active scaffold) is a three dimensional architecture fabricated from intuitive composite materials and able to deliver stimuli perceived as biologically suitable by progenitor cells independently of the presence of soluble biologically active factors, such as growth factors, ligands, etc. IBAS is not a mere mechanical support for cells and bio-active proteins, but concurs with soluble factors to sustain the signals symmetry driving the progenitor cell fate

with the scaffolds forming smaller aggregates, and so increasing cell-substrate rather than cell-cell contact, probably leading to increased expression of ECM and adhesion proteins. An important aspect of the mechano-structural environment is the chemical nature of the substrate, that is the nature of the biomaterial used as an interface for cell adhesion and a structural support for tissues. Recent work has demonstrated that biomaterials, i.e. matrices, scaffolds, and culture substrates, can present key regulatory signals to create artificial surrogate microenvironments that control stem cell fate. Although little is known about the influence of specific biomaterial features, factors, such as ligand density, and material mechanical properties have also been show to play a role in determining phenotype [22, 24]. We would argue that the biomaterial cue belongs to the mechano-structural axis because it is intimately bound with the elastic modulus, although, if the biomaterial is degradable, it can be dramatically modified by the cells themselves, as discussed in the following section. A number of reviews listing the different biomaterials used in stem cell engineering in general are available [11, 30, 63]. However, very little comparative information can be found regarding the performance and effect of materials on stem cell differentiation, since most approaches use a single material and then test various inducing media to assess the differentiating stimulus provided by the material.

In the context of biomaterials for cardiac tissue engineering, probably the best studied mechano-structural feature is the elastic modulus or stiffness of a substrate or scaffold and its effect on the lineage specification. Engler et al. [22] have pioneered studies on matrix elasticity and its effect on mesenchymal stem cells (MSC). Through the use of a well defined elastically tunable polyacrylamide gel (nominally 2D since cells did not penetrate the substrate but adhered on the surface), they observed lineage specification independent of inductive biochemical factors. Softer gels $(0.1-1 \mathrm{kPa})$ were neurogenic, the hardest $(24-40 \mathrm{kPa})$ were 
osteogenic, while gels with intermediate elastic moduli $(8-17 \mathrm{kPa})$ were myogenic. In all three cases the elastic modulus matches that of the corresponding native tissue [21]. Studies conducted on substrates with different stiffness show that in cardiac myocytes the development of aligned sarcomers and stress fibers is higher on substrates with elastic moduli of approximately $10 \mathrm{kPa}$ [32]. In other reports, the range of elastic moduli suitable for sustaining cardiac morphology and function of neonatal rat cardiomyocytes was found to be around $22-50 \mathrm{kPa}$ [8]. Therefore, gel-like materials with elastic moduli between 5 and $50 \mathrm{kPa}$ are most suitable for cardiac tissue engineering, although most studies home into the native elastic modulus of normal tissue which is around 10-20 kPa.

The potency of scaffold stiffness and topology in driving cardiac stem cell differentiation in a three-dimensional culture context was confirmed by Forte et al. [24]. Cardiac stem cells adopted the cardiomyocytic phenotype only when cultured in strictly controlled conditions characterized by a critical combination of chemical, biochemical and physical factors, and emulating the inner myocardial environment. In these studies, the emulation of myocardial environment was achieved by fine-tuning the array of growth factors solved in the culture medium and, above all, the chemistry, topology and stiffness of three-dimensional supports on which stem cells were seeded. The absence of one or more appropriate growth factors or the presence of a polymeric scaffold with stiffness higher than that passively expressed by myocardium did not trigger the differentiating cascade leading to the cardiomyocytic phenotype. Here, scaffold stiffness was modulated by changing the topology of the structure, using a rapid prototyping technique known as PAM (Pressure Assisted Microsyringe, [71]. The optimal mechanical properties to induce cardiomyocyte differentiation was a scaffold stiffness $<30 \mathrm{kPa}$ on scaffolds with square pores of about 100-150 $\mu \mathrm{m}$ [24].

Shear stress induced by flow is also an important physical stimulus, particularly for driving cells towards vascular phenotypes. Flow at physiological wall shear stresses typical of blood vessels $(0.1-1.5 \mathrm{~Pa})$ induces differentiation towards endothelial-like characteristics [51, 72, 78]. Adamo et al. [1] demonstrated that shear stresses of the order of $0.5 \mathrm{~Pa}$ increase haematopoietic colony-forming potential and expression of haematopoietic markers in mouse embryos. Therefore, fluid shear stresses also influences the differentiation pathways of cells indirectly associated with the vascular system. Like scaffold stiffness, wall shear stress is a parameter which can be easily controlled and modulated using engineering design tools and technology.

\section{Current Material-Based Cardiac Regeneration Strategies}

In the past 20 years or so, a number of methods and materials have been employed in an attempt to generate neo-cardiac tissue in vitro as well as in vivo. Traditionally, tissue engineering (TE) strategies to create systems able to replace or repair damaged tissues employ a "top-down" or a "bottom-up" approach as 
Table 2 The top-down and bottom up approaches to engineering the cell microenvironment

\begin{tabular}{|c|c|c|c|}
\hline \multicolumn{2}{|l|}{ Top-down } & \multicolumn{2}{|l|}{ Bottom-up } \\
\hline Methods & Micro stimulus & Methods & Macro construct \\
\hline $\begin{array}{l}\text { Surface } \\
\text { chemistry }\end{array}$ & $\begin{array}{l}\text { Laminin [28], fibronectin [27], } \\
\text { RGD [44] }\end{array}$ & $\begin{array}{l}\text { Micro- } \\
\text { molding }\end{array}$ & $\begin{array}{l}\text { Microwell templates [33] } \\
\text { microchannels [35] }\end{array}$ \\
\hline $\begin{array}{l}\text { Mechanical } \\
\text { properties }\end{array}$ & Elasticity [21], anisotropy [40] & $\begin{array}{l}\text { Self- } \\
\quad \text { assembling }\end{array}$ & $\begin{array}{l}\text { Self-assemled aggregation } \\
\text { [54] }\end{array}$ \\
\hline $\begin{array}{l}\text { Mechanical } \\
\text { environment }\end{array}$ & $\begin{array}{l}\text { Cyclic strain [9], electrical } \\
\text { stimulation [43] }\end{array}$ & $\begin{array}{l}\text { Digital } \\
\text { printing }\end{array}$ & Direct printing [52] \\
\hline Soluble factor & $\begin{array}{l}\text { Morphogenetic proteins [34], } \\
\text { VEGF [66], morphogenetic } \\
\text { proteins }\end{array}$ & Cell sheet & $\begin{array}{l}\text { Sheet based modules [46], } \\
\text { cell laden hydrogel } \\
\text { [59] }\end{array}$ \\
\hline $\begin{array}{l}\text { Architecture } \\
\text { design }\end{array}$ & Topography and shape [24] & & \\
\hline Communication & Cell-cell contact [74] & & \\
\hline
\end{tabular}

$\underline{\text { Table } 3}$ Materials used in cardiac regeneration

\begin{tabular}{|c|c|c|}
\hline \multicolumn{3}{|c|}{ Materials used in cardiac tissue regeneration } \\
\hline \multicolumn{2}{|l|}{ Synthetic } & \multirow{2}{*}{$\begin{array}{l}\text { Natural } \\
\text { Injectable hydrogel }\end{array}$} \\
\hline Solid & Hydrogel & \\
\hline Poly(glycolic acid) (PGA) [24] & $\begin{array}{l}\text { Poly (ethylene glycol)(PEG) } \\
\text { [37] }\end{array}$ & Fibrin [2] \\
\hline $\begin{array}{l}\text { Poly (L-lactic acid) (PLLA) } \\
\text { [24] }\end{array}$ & $\begin{array}{l}\text { Poly (N-isopropylacrylamide) } \\
\text { (PNIPAAm) }\end{array}$ & Collagen [20] \\
\hline $\begin{array}{l}\text { Poly (lactic-co-glycolic acid) } \\
\text { (PLGA) [24] }\end{array}$ & & Matrigel \\
\hline Polycaprolactone (PCL) [24] & & $\begin{array}{l}\text { Self-assembly protein [59] } \\
\quad \text { alignate [19] }\end{array}$ \\
\hline Poly (urethane) [69] & & Hyaluronic acid (HA) [67] \\
\hline
\end{tabular}

shown in Table 2. These methods aim to recreate the complexity of the biological tissues from two different points of view. The "top-down" strategy attempts to control cell fate modeling the interface between cells and polymeric scaffold to mimic the natural extracellular matrix (ECM); while "bottom-up" approach aims to reproduce biomimetic structures using micromodular blocks built together to recreate larger tissues. Many materials have been studied and characterized using the first strategy starting with synthetic polymers, such as poly(glycolic acid) (PGA), poly(L-lactic acid) (PLLA), poly(lactic-co-glycolic acid) (PLGA), poly(ethylene glycol) (PEG) and polycaprolactone (PCL), or natural derived polymers, such as alginate, hyaluronic acid, collagen, fibrin, etc. Some of the most widely reported materials used for investigating cardiac regeneration are reported in Table 3. Their mechanical properties have been widely investigated and they have been processed with different techniques to reproduce ECM elasticity and deformability. In addition, surface modification techniques have been introduced to immobilize specific factors onto the material surfaces to control cell behavior 
Table 4 Commonly used microfabrication techniques for cardiac engineering

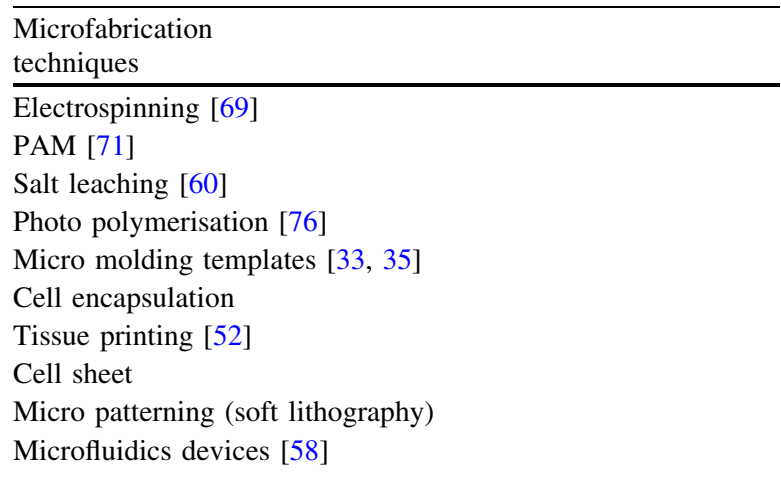

and fate. A key role in designing and realizing a scaffold able to mimic the complexity of the ECM is played by the fabrication technique, some of which are reported in Table 4. In general, the fabrication technique should preserve the properties of the processed material and be capable of producing scaffolds with controlled dimensions (at the nano- and micro-scale) and topology (architecture and shape), as well as high porosity. In the "bottom-up" approach different micromodular structures are designed and built to reproduce tissue engineered constructs with physiological microarchitectural features, providing guidance for tissue morphogenesis at the cellular level. Basically the modules can be created with different microfabrication methods (Table 4) and then assembled randomly or stacked layer-by-layer.

So far, few attempts to reproduce the natural environment for the maintenance and regulation of cardiac stem cells have been carried out. Tissue fabrication techniques have improved a great deal in the past 2 decades and scaffolds can be used to finely tune the chemistry and micro-architecture at the interface between biopolymers and CPC. In particular laminin, fibronectin or RGD sequences can be linked to polymer chains to reproduce ECM signaling allowing precise control of CPC proliferation, survival, differentiation, adhesion and organization. As discussed in Sect. 5, a parameter which can be easily tuned is the elasticity and deformability of scaffolds. These properties are related to the material used, the fabrication process, the porosity and the final scaffold topology. In the case of synthetic polyesters, the porosity, dimensions and topology of scaffold are the key parameters which define mechanical properties, while in hydrogels the crosslinking agent and density as well as polymer characteristics are responsible for material elasticity and stiffness. In fact cell response to substrate stiffness can be elegantly decoupled from biochemical factors using tunable materials such as polyacrylamide or PEG gels thus making it possible to assess which portion of cellular behavior is attributable to cell mechanics [55]. But, while the static mechanical properties of the scaffolds can be easily tuned, the dynamical remodeling of these properties depends on CPC responses, and calls for more responsive materials [65]. 
There is clearly however a synergy between mechanical and chemical cues as demonstrated by Pagliari et al. [57] and Jacot et al. [31] and reviewed by Tenney and Discher [70]. Pagliari and co-workers demonstrated that biological and mechanical signals must cooperate to drive cardiac stem cell differentiation. CPCs only expressed $\alpha$-actinin and displayed sarcomeric bands in the presence of PLA scaffolds with an elastic modulus of $<30 \mathrm{kPa}$ as well as neonatal cardiomyoctes, but not when only one of the two signals were absent. Jacot et al. investigated the correlation between substrate stiffness and chemical pathways. Cells on substrates with the same elastic modulus as healthy cardiac tissue $(10 \mathrm{kPa})$ develop aligned sarcomeres whereas stiffer substrates typified by a fibrotic scar induce differentiation towards a more myoblastic phenotype with long, large stress fibers and low contractile forces. The researchers demonstrated that blocking the RhoA/ROCK pathway, which controls calcium transients during contraction, cell traction on all substrates was significantly increased. The effect of substrate stiffness was therefore overruled by inhibiting the formation of actin stress fibers. It is evident however that substrate stiffness and tension driven matrix assembly are intricately linked as protein function is conditioned by form, which may change during deformation. A clear example is the unfolding of the latent TGF- $\beta 1$ complex, which when activated through stress, releases TFG- $\beta 1$ and induces the initiation of cell contractile machinery which can result in the formation of fibrous stiff tissue.

In the context of cardiac tissue engineering, the "bottom-up" approach can be used to control stem cell response and cell-to-cell electrophysiological interactions using microscale technologies, such as surface topography and micropatterning, or other methods listed in Table 4. Despite the developments in materials science and processing, however, we are still a long way away from identifying a set of materials and rules for processing them for cardiac stem cell maintenance and differentiation. Here too, a paradigm shift, which focuses on biomimetics, complexity and smart, intuitive materials is necessary. In the following section we discuss how to address complexity by fabricating inherently bio-active scaffolds (IBAS).

\section{Smart Materials Strategies: The IBAS Concept}

Electronic and information technologies have made leaps and bounds as far as down-sizing, multiplexing and processing rates are concerned. In stark contrast, technologies which involve interfacing with the biological world are far behind. Materials interfacing with the human body in any context-be it chemical sensors for biological analytes, systems for drug testing or biomaterials-have made remarkably little progress in comparison with say silicon technology. The list of materials approved for in vivo use has remained practically unchanged for decades. Biodegradable polyesters continue to be proposed for tissue engineering applications despite reports on undesired inflammatory response and mechanical failure simply because no viable alternatives are available. 
Currently, biomaterials are prepared for cell adhesion by the addition of adhesion ligands as proteins or peptides. Let us imagine what happens when cells interact with a biomaterial in vitro; the cells find themselves in an environment which besides the presence of adhesion ligands, possesses zero responsiveness. There is a constant tug-of-war between cells and scaffold, usually with the cells losing out. They either give up and die, or secrete a large quantity of adhesive proteins which act as a barrier between the cells and the material, and the cells go it alone. The material on the other hand has remained passive and unresponsive. How would a responsive material behave? How can we make a material respond to its environment by endowing it with intuition?

In our opinion, to answer the questions, one of the key factors that must be addressed is the complexity of the human body and the interactions between different organs, systems and tissues, as well as the profound role the environment and external chemical or physical factors have on the body's response. No material in the human body is monocomponent and all senses-be they chemical or physical-are integrated, adaptive and fuzzy. Moreover, the materials in our bodies are intuitive, because they react to cues from their environment, and they do so because sensing is an integral part of their structure. Taking a cue from biology, or using the much abused term "biomimetics", the complexity must be challenged and met with complex materials and sensing systems which exploit the plethora of knowledge acquired in chemistry and physics to create new hybrid composite materials and alternative transduction modalities. These materials then need to be shaped into three dimensional scaffolds to produce what we call inherently bioactive scaffolds, or IBAS. IBAS are scaffolds which are able to mimic the tissue microenvironment and address stem cell adhesion, growth and differentiation in the absence of biological factors or any biological functionalization. To emulate tissue complexity, IBAS should be composed of interconnected modules having distinct features, which could be recognized by $\mathrm{CPC}$ at the nano- and microscale level (Fig. 4).

Until now, the cell has been considered as an entity doing its job independently from the others, and from the scaffold or substrate, which is unrealistic in vivo. In vivo, not only is the cell in equilibrium with its neighbors, but also with the extracellular matrix. Moreover, the matrix itself is in equilibrium with the cells as it too responds to and is modified by the cells. Therefore, each cell's strategy, as well as the matrix's can be independent, but the overall result will lead to an equilibrium state. Using this hypothesis, an IBAS could be engineered to coerce the system towards an equilibrium consisting of myocardial tissue.

Some attempts have been made to create complex material systems, although these are often based on mixtures of polymers [13]. Carbon nanotubes are also becoming popular additives to classic biomaterials [47], but their main function is to increase the elastic modulus rather than confer sensing or other properties. However, as Whulanza et al. [75] show, CNT loaded scaffolds can sense cellular behavior through changes in the electrical properties of the material. Other, more ambitious, solutions could be explored such as that reported by [73] who developed a water and nanoparticle based hydrogel which has the strength to remain self 
supporting, as well as self-repairing. Other methods to render scaffolds intuitive involve integration of nanosenors into the materials, either during processing [15] or synthesis [81]. On the other hand, as suggested and demonstrated by Schneider and Strongin [65], polymers can even be designed so that a particular chemical substance or supramolecular binding site reacts selectively, reversibly and noncovalently to change the properties of the polymer, even at a local scale. In the majority of cases, the changes are conformational, and based on the exclusion or inclusion of water molecules. This is exactly how sensors in biology work.

\section{Conclusion}

In conclusion, materials need to be rethought, reshaped and reassembled using a bottom-up reverse engineering approach, in which the end goal is a purpose or a function irrespective of composition. New approaches should consider the mechanical, chemical and topological signals and design new complex multi-shell [79] or nanocomposite materials able to encapsulate cells, which not only allow induction or control of cell fate, but also enable dynamic remodeling of the cell microenvironment. Using intuitive and adaptive complex materials, or IBAS, different microsystems can be assembled into a modular engineered tissue. The IBAS can be thought of as an intuitive scaffold which naturally leads CPC through a set of equilibrium states, ending with functional cardiac tissue.

\section{References}

1. Adamo, L., Naveiras, O., Wenzel, P.L., et al.: Biomechanical forces promote embryonic haematopoiesis. Nature 459, 1131-1135 (2009)

2. Ahmed, T.A., Dare, E.V., Hincke, M.: Fibrin: a versatile scaffold for tissue engineering applications. Tissue Eng. Part B 14, 199-215 (2008)

3. Andersen, D.C., Andersen, P., Schneider, M., et al.: Murine "cardiospheres" are not a source of stem cells with cardiomyogenic potential. Stem Cells 27, 1571-1581 (2009)

4. Anversa, P., Kajstura, J., Leri, A., et al.: Life and death of cardiac stem cells: a paradigm shift in cardiac biology. Circulation 113, 1451-1463 (2006)

5. Baharvand, H., Hashemi, S.M., Ashtiani, S.M., et al.: Differentiation of human embryonic stem cells into hepatocytes in $2 \mathrm{D}$ and $3 \mathrm{D}$ culture systems in vitro. Int. J. Dev. Biol. 50, 645-652 (2006)

6. Beltrami, A.P., Cesselli, D., Bergamin, N., et al.: Multipotent cells can be generated in vitro from several adult human organs (heart, liver, and bone marrow). Blood 110, 3438-3446 (2007)

7. Bergmann, O., Bhardwaj, R.D., Bernard, S., et al.: Evidence for cardiomyocyte renewal in humans. Science 324, 98-102 (2009)

8. Bhana, B., Iyer, R.K., Chen, W.L.K., et al.: Influence of substrate stiffness on the phenotype of heart cells. Biotechnol. Bioeng. 105(6), 1148-1160 (2010)

9. Boublik, J., Park, H., Radisic, M., et al.: Mechanical properties and remodelling of hybrid cardiac constructs made from heart cells, fibrin, and biodegradable, elastomeric knitted fabric. Tissue Eng. 11, 1122-1132 (2005) 
10. Brännvall, K., Bergman, K., Wallenquist, U., et al.: Enhanced neuronal differentiation in a three-dimensional collagen-hyaluronan matrix. J. Neurosci. Res. 85, 2138-2146 (2007)

11. Burdick, J.A., Vunjak-Novakovic, G.: Engineered microenvironments for controlled stem cell differentiation. Tissue Eng. Part A 15, 205-219 (2009)

12. Chen, S.L., Fang, W.W., Ye, F., et al.: Effect on left ventricular function of intracoronary transplantation of autologous bone marrow mesenchymal stem cell in patients with acute myocardial infarction. Am. J. Cardiol. 94, 92-95 (2004)

13. Chiono, V., Pulieri, E., Vozzi, G., et al.: Genipin-crosslinked chitosan/gelatin blends for biomedical applications. J. Mater. Sci. Mater. Med. 19, 889-898 (2008)

14. Claycomb, W.C., Di Nardo, P. (eds.): Cardiac Growth and Regeneration, vol. 752. New York Academy of Sciences, New York (1995)

15. Coupland, P.G., Briddon, S.J., Aylott, J.W.: Using fluorescent pH-sensitive nanosensors to report their intracellular location after Tat-mediated delivery. Integr. Biol. 1, 318-323 (2009)

16. Cross, M.A., Enver, T.: The lineage commitment of haemopoietic progenitor cells. Curr. Opin. Genet. Dev. 7, 609-613 (1997)

17. Dawn, B., Stein, A.B., Urbanek, K., et al.: Cardiac stem cells delivered intravascularly traverse the vessel barrier, regenerate infarcted myocardium, and improve cardiac function. Proc. Natl. Acad. Sci. USA 102, 3766-3771 (2005)

18. Di Nardo, P., Forte, G., Ahluwalia, A., et al.: Cardiac progenitor cells: potency and control. J. Cell. Physiol. 224, 590-600 (2010)

19. Drury, J.L., Dennis, R.G., Mooney, D.J.: The tensile properties of alginate hydrogels. Biomaterials 25, 3187-3199 (2004)

20. Eghbali, M., Weber, K.T.: Collagen and the myocardium: fibrillar structure, biosynthesis and degradation in relation to hypertrophy and its regression. Mol. Cell. Biochem. 96, 1-14 (1990)

21. Engler, A.J., Carag-Krieger, C., Johnson, C.P., et al.: Embryonic cardiomyocytes beat best on a matrix with heart-like elasticity: scar-like rigidity inhibits beating. J. Cell Sci. 121, 3794-3802 (2008)

22. Engler, A.J., Sen, S., Sweeney, H.L., et al.: Matrix elasticity directs stem cell lineage specification. Cell 126, 677-689 (2006)

23. Fischer-Rasokat, U., Assmus, B., Seeger, F.H., et al.: A pilot trial to assess potential effects of selective intracoronary bone marrow-derived progenitor cell infusion in patients with nonischemic dilated cardiomyopathy: final 1-year results of the transplantation of progenitor cells and functional regeneration enhancement pilot trial in patients with nonischemic dilated cardiomyopathy. Circ. Heart Fail. 2, 417-423 (2009)

24. Forte, G., Carotenuto, F., Pagliari, F., et al.: Criticality of the biological and physical stimuli array inducing resident cardiac stem cell determination. Stem Cells 26, 2093-2103 (2008)

25. Fuchs, E., Tumbar, T., Guasch, G.: Socializing with the neighbors: stem cells and their niche. Cell 116, 769-778 (2004)

26. Gaetani, R., Ledda, M., Barile, L., et al.: Differentiation of human adult cardiac stem cells exposed to extremelylow-frequencies electromagnetic fields. Cardiovasc. Res. 82, 411-420 (2009)

27. Gelain, F., Bottai, D., Vescovi, A., et al.: Designer self-assembling peptide nanofiber scaffolds for adult mouse neural stem cell 3-dimensional cultures. PLoS ONE 1, e119 (2006)

28. Genove, E., Shen, C., Zhang, S., et al.: The effect of functionalised self-assembling peptide scaffolds on human aortic endothelial cell function. Biomaterials 26, 3341-3351 (2005)

29. Hare, J.M., Traverse, J.H., Henry, T.D., et al.: A randomized, double-blind, placebocontrolled, dose-escalation study of intravenous adult human mesenchymal stem cells (prochymal) after acute myocardial infarction. J. Am. Coll. Cardiol. 54, 2277-2286 (2009)

30. Hwang, N.S., Varghese, S., Elisseeff, J.: Controlled differentiation of stem cells. Adv. Drug Deliv. Rev. 60, 199-214 (2008)

31. Jacot, J.G., Kita-Matsuo, H., Wei, K.A., et al.: Cardiac myocyte force development during differentiation and maturation. Ann. NY Acad. Sci. 1188, 121-127 (2010) 
32. Jacot, J.G., McCulloch, A.D., Omens, J.H.: Substrate stiffness affects the functional maturation of neonatal rat ventricular myocytes. Biophys. J. 95, 3479-3487 (2008)

33. Karp, J.M., Yeh, J., Eng, G., et al.: Controlling size, shape and homogeneity of embryoid bodies using poly(ethylene glycol) microwells. Lab Chip 7, 786-794 (2007)

34. Keegan, B.R., Feldman, J.L., Begemann, G., et al.: Retinoic acid signalling restricts the cardiac progenitor pool. Science 307, 247-249 (2005)

35. Khademhosseini, A., Eng, G., Yeh, J., et al.: Microfluidic patterning for fabrication of contractile cardiac organoids. Biomed. Microdevices 9, 149-157 (2007)

36. Laugwitz, K.L., Moretti, A., Lam, J., et al.: Postnatal is $11^{+}$cardioblasts enter fully differentiated cardiomyocyte lineages. Nature 433, 647-653 (2005)

37. Leslie-Barbick, J.E., Moon, J.J., West, J.L.: Covalently-immobilized vascular endothelial growth factor promotes endothelial cell tubulogenesis in poly(ethylene glycol) diacrylate hydrogels. J. Biomater. Sci. 20, 1763-1779 (2009)

38. Levenberg, S., Huang, N.F., Lavik, E., et al.: Differentiation of human embryonic stem cells on three-dimensional polymer scaffolds. Proc. Natl. Acad. Sci. 100, 12741-12746 (2003)

39. Li, L., Xie, T.: Stem cell niche: structure and function. Annu. Rev. Cell Dev. Biol. 21, 605-631 (2005)

40. Linask, K.K., Han, M., Cai, D.H., et al.: Cardiac morphogenesis: matrix metalloproteinase coordination of cellular mechanism underlying heart tube formation and directionality of looping. Dev. Dyn. 233, 739-753 (2005)

41. Liu, H., Lin, J., Roy, K.H.: Effect of 3D scaffold and dynamic culture condition on the global gene expression profile of mouse embryonic stem cells. Biomaterials 27, 5978-5989 (2006)

42. Liu, H., Roy, K.: Biomimetic three-dimensional cultures significantly increase hematopoietic differentiation efficacy of embryonic stem cells. Tissue Eng. 11, 319-330 (2005)

43. Ma, W., Liu, Q.Y., Jung, D., et al.: Central neuronal synapse formation on micropatterned surfaces. Brain Res. Dev. Brain Res. 111, 231-243 (1998)

44. Mann, B.K., Gobin, A.S., Tsai, A.T., et al.: Smooth muscle cell growth in photopolymerised hydrogels with cell adhesive and proteolytically degradable domains: synthetic ECM analogs for tissue engineering. Biomaterials 22, 3045-3051 (2001)

45. Martin, C.M., Meeson, A.P., Robertson, S.M., et al.: Persistent expression of the ATP-binding cassette transporter, Abcg2, identifies cardiac SP cells in the developing and adult heart. Dev. Biol. 265, 262-275 (2004)

46. Masuda, S., Shimizu, T., Yamato, M., et al.: Cell sheet engineering for heart tissue repair. Adv. Drug Del. Rev. 60, 277-285 (2008)

47. Mattioli-Belmonte, M., Vozzi, G., Seggiani, M., et al.: Tuning polycaprolactone-carbon nanotube composites for bone tissue engineering scaffolds. Mater. Sci. Eng. Biomimetic Mater. (2010, in press)

48. Matsuura, K., Nagai, T., Nishigaki, N, et al.: Adult cardiac Sca-1-positive cells differentiate into beating cardiomyocytes. J. Biol. Chem. 279, 11384-11391 (2004)

49. Menasché, P.: Cell therapy: results in cardiology. Bull. Acad. Natl. Med. 193, 559-568 (2009)

50. Messina, E., De Angelis, L., Frati, G., et al.: Isolation and expansion of adult cardiac stem cells from human and murine heart. Circ. Res. 95, 911-921 (2004)

51. Metallo, C.M., Vodyanik, M.A., de Pablo, J.J., et al.: The response of human embryonic stem cell-derived endothelial cells to shear stress. Biotechnol. Bioeng. 100, 830-837 (2008)

52. Mironov, V., Boland, T., Trusk, T., et al.: Organ printing: computer-aided jet-based 3D tissue engineering. TRENDS Biotechnol. 21, 157-161 (2003)

53. Murry, C.E., Soonpaa, M.H., Reinecke, H., et al.: Haematopoietic stem cells do not transdifferentiate into cardiac myocytes in myocardial infarcts. Nature 428, 664-668 (2004)

54. Napolitano, A.P., Chai, P., Dean, D.M., et al.: Dynamics of the self-assembly of complex cellular aggregates on micromolded nonadhesive hydrogels. Tissue Eng. 13, 2087-2094 (2007)

55. Nemir, S., West, J.L.: Synthetic materials in the study of cell response to substrate rigidity. Ann. Biomed. Eng. 38, 2-20 (2010) 
56. Oh, H., Bradfute, S.B., Gallardo, T.D., et al.: Cardiac progenitor cells from adult myocardium: homing, differentiation, and fusion after infarction. Proc. Natl. Acad. Sci. USA 100, 12313-12318 (2003)

57. Pagliari, S., Vilela-Silva, A.C., Forte, G.C., Pagliari, F., Mandoli, C., Vozzi, G., Pietronave, S., Prat, M., Licoccia, S., Ahluwalia, A., Traversa, E., Minieri, M., Di Nardo, P., Cooperation of biological and mechanical signals in cardiac progenitor cell differentiation. Adv. Mater. (2010) 12 Nov 2010. doi:10.1002/adma.201003479.

58. Paguirigan, A.L., Beebe, D.J.: Protocol for the fabrication of enzymatically crosslinked gelatin microchannels for microfluidic cell culture. Nat Protocols 2, 1782-1788 (2007)

59. Palmer, L.C., Stupp, S.I.: Molecular self-assembly into one-dimensional nanostructures. Acc. Chem. Res. 41, 1674-1684 (2008)

60. Pego, A.P., Siebum, B., Van Luyn, M.J., et al.: Preparation of degradable porous structures based on 1, 3-trimethylene carbonate and D,L-lactide (co)polymers for heart tissue engineering. Tissue Eng. 9, 981-994 (2003)

61. Reilly, G.C., Engler, A.J.: Intrinsic extracellular matrix properties regulate stem cell differentiation. J. Biomech. 43, 55-62 (2010)

62. Rosenblatt-Velin, N., Lepore, M.G., et al.: FGF-2 controls the differentiation of resident cardiac precursors into functional cardiomyocytes. J. Clin. Invest. 115, 1724-1733 (2005)

63. Saha, K., Pollock, J.F., Schaffer, D.V., et al.: Designing synthetic materials to control stem cell phenotype. Curr. Opin. Chem. Biol. 11, 381-387 (2007)

64. Scadden, D.T.: The stem-cell niche as an entity of action. Nature 441, 1075-1079 (2006)

65. Schneider, H.J., Strongin, R.M.: Supramolecular interactions in chemomechanical polymers. Acc. Chem. Res. 42, 1489-1500 (2010)

66. Seliktar, D., Zisch, A.H., Lutolf, M.P., et al.: MMP-2 sensitive, VEGF-bearing bioactive hydrogels for promotion of vascular healing. J. Biomed. Mater. Res. A 68, 704-716 (2004)

67. Serban, M.A., Prestwich, G.D.: Modular extracellular matrices: solutions for the puzzle. Methods 45, 93-98 (2008)

68. Smith, R.R., Barile, L., Cho, H.C., et al.: Regenerative potential of cardiosphere-derived cells expanded from percutaneous endomyocardial biopsy specimens. Circulation 115, 896-908 (2007)

69. Stankus, J.J., Guan, J., Fujimoto, K., et al.: Microintegrating smooth muscle cells into a biodegradable, elastomeric fiber matrix. Biomaterials 27, 735-744 (2006)

70. Tenney, R.M., Discher, D.E.: Stem cells, microenvironment mechanics, and growth factor activation. Curr. Opin. Cell Biol. 21, 630-635 (2009)

71. Vozzi, G., Previti, A., De Rossi, D., et al.: Microsyringe-based deposition of two-dimensional and three-dimensional polymer scaffolds with a well-defined geometry for application to tissue engineering. Tissue Eng. 8, 1089-1098 (2002)

72. Wang, H., Riha, G.M., Yan, S., et al.: Shear stress induces endothelial differentiation from a murine embryonic mesenchymal progenitor cell line. Arterioscler. Thromb. Vasc. Biol. 25, $1817-1823$ (2005)

73. Wang, Q., Mynar, J.L., Yoshida, M., et al.: High-water-content mouldable hydrogels by mixing clay and a dendritic molecular binder. Nature 463, 339-343 (2010)

74. White, S.M., Claycomb, W.C.: Embryonic stem cells form an organised, functional cardiac conduction system in vitro. Am. J. Physiol. Heart Circ. Physiol. 288, H670-H679 (2005)

75. Whulanza, Y., Uccifferri, N., Vozzi, G., Domenici, C.: Sensing scaffolds to monitor cellular activities using impedance measurements. Biosensors Bioelectronics (in press)

76. Williams, C.G., Malik, A.N., Kim, T.K., et al.: Variable cytocompatibility of six cell lines with photoinitiators used for polymerising hydrogels and cells encapsulation. Biomaterials 26, 1211-1218 (2005)

77. Wollert, K.C., Meyer, G.P., Lotz, J., et al.: Intracoronary autologous bone-marrow cell transfer after myocardial infarction: the BOOST randomised controlled clinical trial. Lancet 364, 141-148 (2004) 
78. Wu, C.C., Chao, Y.C., Chen, C.N., et al.: Synergism of biochemical and mechanical stimuli in the differentiation of human placenta-derived multipotent cells into endothelial cells. J. Biomech. 41, 813-821 (2008)

79. Yao, H., Dao, M., Imholt, T., et al.: Protection mechanisms of the iron-plated armor of a deep-sea hydrothermal vent gastropod. Proc. Natl. Acad. Sci. USA 107, 987-992 (2010)

80. Zampetaki, A., Kirton, J.P., Xu, Q.: Vascular repair by endothelial progenitor cells. Cardiovasc. Res. 78, 413-421 (2008)

81. Zhang, G., Palmer, G.M., Dewhirst, M.W., et al.: A dual-emissive-materials design concept enables tumour hypoxia imaging. Nat. Mater. 8, 747-751 (2009)

82. Zipori, D.: The stem state: plasticity is essential, whereas self-renewal and hierarchy are optional. Stem Cells 23, 719-726 (2005) 